\title{
The Study on the Nursing Intervention of the Pedicle Flap Transposition Repairing Traumatic Tissue Defect in the Hands
}

\author{
Ning $\mathrm{Jia}^{1, \text { a }}$,Xiaoting Zhou ${ }^{2, *}$,Fenge Wang $^{3}$, Yeben Wang ${ }^{4}$, Yan Zhao ${ }^{4}$, \\ Jian Wang ${ }^{4}$ \\ ${ }^{1}$ Department of hand Surgery, Third People's Hospital of Jinan, Jinan, 250132, China. \\ ${ }^{2}$ Department of gynecology, Third People's Hospital of Jinan, Jinan, 250132, China. \\ ${ }^{3}$ Department of Emergency Medicine, Third People's Hospital of Jinan, Jinan, 250132, China. \\ ${ }^{4}$ Department of hand Surgery, Third People's Hospital of Jinan, Jinan, 250132, China. \\ aningjia666@163.com
}

*Corresponding author wang578@163.com

\section{Keywords: Pedicle flap; Repair; Hand trauma; Tissue defect}

\begin{abstract}
Objective: To explore hand traumatic tissue defect in the microsurgical repair methods and their effects. Methods: We use the index finger dorsal island flap, stitched nerve dorsal finger artery retrograde island flap, adjacent finger flap, dorsal metacarpal artery retrograde island flap and forearm interosseous artery after retrograde iliac groin flap, flap, iliac flap and umbilical thoracic flap transposition to repair hand traumatic tissue defect in 120 cases, and nursing intervention, the treatment effect and functional recovery were observed. Results: The tissue flap all survived, and followed up for 3 months to 1 year, stomach feel through your fingers, S2 - pd: 5 to $7 \mathrm{~mm}$, can maximum limit retained and the restoration of hand function and appearance. Conclusion: The pedicle flap transposition repairing traumatic tissue defect in hands, effect is satisfactory.
\end{abstract}

\section{Introduction}

The hand trauma is rare, especially finger skin defect after trauma, leg muscle, bones, joints and exposed nerves, blood vessels, is a common clinical. The hand defects, especially with tendons, joint composite tissue defects, such as clinical treatment more difficult. With the continuous development of microsurgical technique, tissue flap transplantation to repair tissue defect of the body has become a kind of effective treatment, makes possible the success of the past is difficult to repair the wound repair. The application of pedicle skin flap transplantation to repair tissue defect received very good clinical effect. With vascular pedicle flap translocation is the nutrient vessels of the flap, through local transfer of adjacent tissue repair surgery, rotating arc, it to flap covering the defect area is wide and effective solution to this problem. Choose between February 2013 and October 2014 the application of our hospital with pedicled flap transposition to repair traumatic tissue defect in 120 cases of the nursing experience of summary reports as follows.

\section{Materials and Methods}

We selected from February 2013 to October 2014, our hospital hand traumatic tissue defect in 120 cases of patients, 78 cases of male, female 42 cases; Aged $6 \sim 78$, an average of 26.8 years. Causes: machine (hot) 96 cases, pressure parts in 16 cases, chainsaw 8 cases. Injury: thumb abdominal defect in 12 cases, 6 cases index finger tip defect, middle finger defect in 5 cases, 6 cases of ring finger tip defect of index finger section volar skin defect in 7 cases, ring refers to the section of 8 cases of volar skin defect, the index finger, nearly section refers to the dorsal skin defect in 9 cases, middle finger, nearly section refers to the dorsal skin defect in 6 cases, ring finger section refers to the dorsal skin defect in 8 cases, $2 \sim 4$ refers to the volar skin and segmental defect tendons exposed in 2 cases, dorsal skin and segmental defect in 2 cases; The back of the hand skin defect, 
including 13 cases of extensor tendon exposed, 10 cases of the skin defect of neurovascular palm tendon exposed; Skin to take off the set of thumb injury and 8 cases of skin defect of the hand, tucks, thumb defect and the back of the hand skin defect in 4 cases, more refers to the skin to take off the set of 14 cases of large skin defects and hand back. Skin defect area: $2.5 \mathrm{~cm} * 3 \mathrm{~cm} \sim 8 \mathrm{~cm} *$ $25 \mathrm{~cm}$; Trauma to repair time $4 \sim 8 \mathrm{~h}$.

\section{The methods}

The routine after thorough debridement is according to the defects of the hand wound site, area, repairing function requirement and local blood vessel condition, taking corresponding selection, design, and the flap transposition to repair. Including: (1) the index finger dorsal island flap repair of the thumb by transfer of finger pulp defect 16 cases; (2) stitched indicated nerve dorsal branch artery retrograde island flap transposition to repair, ring finger end defects in food, 20 cases; (3) adjacent refers to food, skin flap transposition repair ring refers to the volar skin defect 15 cases; (4) the dorsal metacarpal artery retrograde island flap transposition to repair, in food, ring finger dorsal skin defect 20 cases; (5) after forearm interosseous artery retrograde island flap to repair the skin defect of hand or palm 17 cases; (6) spin iliac shallow vascular pedicle iliac groin flap bridge cross transposition repairing segmental pointed to dorsal or volar skin defect in 5 cases, 12 cases to repair the skin defect in the back of the hand or hands, skin repair thumb finger and hand back, tucks 8 cases of skin defect; (7) spin iliac shallow vascular bridge cross transposition flap reconstruction thumb defect and repair of hand skin defect back 7 cases; Being umbilical branch hose chest thoracic flap bridge cross transposition to repair the skin more to take off the set of 12 cases and the back of the hand skin defect.

\section{The care methods}

\section{The preoperative nursing care}

For the skin area body ban puncture or injection, to observe the local presence of furuncle swollen, scar, etc. Preoperative skin preparation operation area is larger, should across more than two joints, such as remove abdominal tissue flap to shaving the pubic hair, shaved so must brachial plexus anesthesia with side armpit hair, introduce the operation situation and treatment effect to the patient at the same time, to alleviate tension and anxiety in patients with psychological. Especially the postoperative body to be connected to for the skin area 20 days or so, might bring inconvenience to get their cooperation.

\section{The postoperative care}

(1) The general nursing

Postoperative put patients in air-conditioned room, bed cotton-padded mattress should not be high, a strong support bed, bed of avoid by all means when patients in order to prevent dehiscence. Tell patients into high protein, high quantity of heat diet, in order to promote the body recovery. Assist the patient to finish the dress, wash care.

(2) The fixed body

The different flap transplantation postoperative position and resettlement, but according to the resettlement principle, hold the hand rest position, flap upward, transplant centers can be exposed, to keep her firmness, avoid reverse. Raise the limb $10^{\circ} \sim 20^{\circ}$, to hand over arm flap, chest, and abdomen flap transplantation with a pillow raised elbow, avoid joints where empty. Can be used within three days the corset or bandage appropriate fixed limb, avoid limb night pull up dramatically. Three days after basic habits in patients with limb put the position, can be fixed to loosen or remove the corset or bandage. Flap and the flap is generally adopt the adjacent finger plaster external fixation. For limb long fixed limb paralysis will appear in the same position, the nurse to assist and guide the family members of limb centripetal massage, to eliminate the paralysis is unwell, improve circulation, avoid limb muscle atrophy. 
(3) The tissue flap observation and treatment

With vascular pedicle flap repair surgery, often should observe tissue flap. Normal tissue flap is ruddy appearance, skin temperature, postoperative by low heats up to the normal or slightly higher than normal. If there is any exception should promptly report to the doctor find out the reason, take corresponding measures, timely processing usually can get satisfactory effect.

(4) The tissue flap appearance of judgment

The tissue reaction has been eliminated, 10 days postoperatively tissue flap with local microcirculation and basic establishment. In order to reduce postoperative flap bloated, especially those with abdominal skin flap transfer operation, can be to gently pull the skin flap, from less to more, from small to large. Force must be observed when the local blood supply, shall not be too hard.

(5) The break time judgment

The early break will affect the quality of tissue flap, too late, the broken causing inconvenience to patients; increase the medical expense. General $18 \sim 21$ days postoperatively, in the test should be taken before broken pedicle skin flap of blood circulation, to ensure the flap transfer success. Clinically often can use rubber band around the flap pedicle, block of blood supply, observe tissue flap blood supply situation, such as can last $1 \sim 2$ hours, the flap is not blue, pale, edema, blood supply and backflow, reliable and safe to break. As the scarlet turned purple, white it need to extend the break time.

(6) The specialist guidance

After anesthesia, with vascular pedicle flap to repair hand wound surgical trauma, and to sacrifice a piece of tissue of the body, combined with the limb should be in a forced postures, cannot optional activities, unstable patients prone to anxiety, irritability, etc. Worry my limbs will bring inconvenience to the life, worry about whether the operation can be successful on the other hand, surgery can achieve satisfactory appearance and function, especially worried about surgery failure will lead to greater disability. Nurse should give psychological counseling in time, make the patient produces a sense of security and belonging and according to the condition change development, and progressive disease related knowledge, appropriate to give rehabilitation guidance. Skin flap after the break, feel is missing in the short term, so before feeling function has yet to recover, to guide the patients anti-scald, frostbite; Refers to the abdominal skin flap can be more friction and normal fingers, in order to promote the sense of touch, and make local skin thickening, wear-resisting.

\section{Results}

The tissue flap all survived, and after $3 \sim 4$ weeks off, points to three months. 2 cases of tendon adhesion to phase ii release, secondary per arthritis of shoulder 8 cases cured by anti-inflammatory analgesic, physical therapy and functional exercise. Follow-up of 3 months to 1 year, stomach feel through your fingers, $\mathrm{S} 2$ - pd $5 \sim 7 \mathrm{~mm}$, all cases can keep maximum and the restoration of hand function and appearance.

\section{Discussion}

\section{The advantages of a traumatic tissue defect repair the hand}

Deep skin defect of hand trauma easily merge blood vessels, nerves, tendons exposed, one tissue flap transposition to repair, to maximize reserve and recovery of hand function and appearance. Its advantages: (1) not because of fear to remove contusion tissue cannot close the wound and too much for palliative treatment, only make debridement more thoroughly, in order to reduce postoperative adhesion and prevent infection, guarantees for tissue repair success; 2 wound clear anatomical structures, and can avoid secondary repair when injured blood vessels, nerve important due to unclear anatomy structure, also is advantageous to the hand function recovery as early as possible; (3) a period to repair wounds, is a prerequisite to success of deep tissue repair, can prevent the secondary repair wound is not healing and mixed infection, exposed tendons, blood vessels, 
nerves, bone necrosis, scar hyperplasia of tendon adhesion and joint contracture caused by hand dysfunction; (4) whether to shorten the duration of symptoms and restore function, or reduce the wounded pain and lower health care costs are helpful. This group of 191 cases were I phase, shaft type flap blood supply is rich, strong appeal, can improve the local blood supply, promote deep tissue repair and wound healing. This group of cases were primary healing, 5 cases of tendon adhesion phase for I flap transposition with good skin condition, adhesion release technique to obtain satisfactory results.

\section{The selection of pedicle flap and operation essentials}

According to the defects of the hand parts need, the size of the wound and repair function, comply with the principle, the preferred hand or forearm and local skin flap pedicle transfer, second bridge cross the hindquarters cadres flap transposition to repair. Index finger dorsal island flap transposition, the index finger dorsal island flap is the first palm dorsal artery blood supply and dorsal metacarpal veins as venous return, and contains the index called lateral dorsal nerve, belong to feel flap for repair of the thumb by transfer of finger pulp defect. Using this flap, 21 cases of successful repair thumb abdominal defect. Operation main points: (1) should be close to the index finger flap design section nearly called lateral side line, make sure it contains the radial side refers to the dorsal artery; The first palm dorsal artery and the dorsal metacarpal veins 2, although not a superficial branch of radial nerve bundle, but walked in the same direction. Flap should be artery, vein and nerve when cut along with subcutaneous tissue during the separation, formation of neurovascular bundle and protection. Free vascular pedicle to radial dorsal joint from 2, stay away from the first palm dorsal artery, with the palm artery traffic, and keep it with radial side refers to the continuity of dorsal artery; (3) The accidentally injured vascular pedicle, or encounter the first palm back side of the terminal branch of the index finger dorsal radial artery is fine and the thumb foot dorsal branch is coarse, need to use forefinger adjacent skin flap to repair.

Dorsal branch of stitched refers to nerve artery retrograde island flap transposition: according to both sides to show a nodal artery horizontal transverse traffic of sex match each other, to refer to distal arterial retrograde blood supply of finger in the proximal lateral finger artery retrograde island flap design, cut skin flap with segmental nerve dorsal branch, inversion and the area near side refers to the nerve end after the bridge, make the skin flap for good feeling. The retrograde flap transfer can repair defect through your fingers. Group adopted the transposition, success at the end of, ring finger food, repair, a total of 30 cases. Operation main points: (1) preoperative using the examination or mean arterial pressure test on both sides, confirmed on both sides of the normal mean artery, can cut the flap; 2 don't drive a tourniquet on blood, to ensure the operation field can clearly show mean artery; (3) with sharp dissection in refers to the artery and nerve refers to the nerve preservation in situ, because the finger artery usually without accompanying vein, the vascular pedicle should try to keep arteries surrounding tissue, also in the superficial fascia superficial vein were cut out, constitute the vascular pedicle, to facilitate postoperative flap blood flowing. Taking throughout and the flap of the segmental nerve dorsal branch, moved to the area it nearly end stitched and refers to the nerve, make the skin flap after obtain good feeling.

Adjacent skin flap transposition: our group USES the side flip adjacent refers to food, skin flap transposition successful repair ring refers to volar, a total of 21 cases of skin defect. The flap is a small artery dry branch vascular flap, namely to refers to the refers to the dorsal branches of the artery blood supply, cut out without being limited by the aspect ratio, but not more than refers to the side of the center line to prevent injury refers to refers to the dorsal branches of the artery. Flap pedicle for not too short, in order to prevent flap transfer after the tension and affect flap blood supply; And slightly longer portable and broken easily shut down when the supply and the district. For skin graft packing pressure is fixed, the power to do not affect skin flap and supply refers to the blood supply.

Dorsal metacarpal artery retrograde island flap transposition: this group by using this flap transposition, successful repair, ring finger dorsal skin defect in food, a total of 28 cases. Dorsal metacarpal artery retrograde island flap with dorsal metacarpal artery and the dorsal metacarpal 
superficial vein as the vascular pedicle, with dorsal metacarpal artery and the palm side of total artery and vein between dorsal metacarpal veins and head for the axis of rotation in refers to the division of web form retrograde blood supply and return of retrograde island flap. Operation main points: (1) surface of deep dorsal metacarpal artery located in the back of hand muscles, constant line up the metacarpal clearance center line to the corresponding interosseous dorsal muscle membrane surface. Note the position of the finger extensor tendon in the hand back often and subject to change with different postures, cut skin flap should be keep in neutral position, in order to make the refers to the extensor tendon in corresponding metacarpal back and show the metacarpal clearance, dorsal metacarpal veins separation. Encounter refers to the extensor tendon combined obstruction of shaft type taking, the separation of the blood vessel can be cut off; 2 dorsal metacarpal artery and the dorsal metacarpal veins are no beam, but walked in the same direction. Flap should be cut arteries and veins along with subcutaneous tissue during the separation, the formation of blood vessel bundle for protection. Free vascular pedicle to joint level refers to web with dorsal metacarpal artery is refers to the side of total artery traffic palmer branch and the dorsal metacarpal veins and venous intersection between head, don't damage as the axis of rotation, to ensure that the flap by the retrograde flow blood supply; (3) taking flap sharpness, be sure to keep skin flap and its connected to loose the superficial fascia and dorsal metacarpal artery between continuity, pay attention to light and don't damage the superficial fascia with fine operation skin vascular, to ensure that the skin flap blood supply; (4) with dorsal metacarpal artery and vein is fine, as long as dorsal metacarpal venous distribution allows, should try to get connected to the flap of dorsal metacarpal veins and venous between head, as a retrograde flap blood flow channel, to prevent postoperative flap blood reflux. Therefore preoperative should observe the distribution of palm-dorsal vein image, in the design to include palm dorsal vein skin flap.

Forearm interosseous artery after retrograde flap transposition: this group by using this flap transposition, success to repair the skin defect of hand or hand, a total of 21 cases. The forearm interosseous dorsal artery flap is after forearm interosseous artery blood supply type axial flap, clearance skin vascular flap. Flap cortical good, moderate thickness, good elasticity, color is in line with the hand, axial type vessels less variation, and for the main blood vessels, taking two main blood vessels without sacrificing the forearm flap. It's vascular pedicle retrograde inversion can repair the backs of your hands, palms wound. Operation main points: (1) the forearm interosseous dorsal blood vessels and the branches are finer, therefore should not drive a tourniquet on blood, make the vascular engorgement coarse is easy to find and anatomy and beneficial to skin flap for success; Taking the retrograde island flap, 2 distal to the free vascular pedicle can't more than 2.5 $\mathrm{cm}$ on the process of the two, so as not to damage the volar between bone vascular of dorsal branch, cause the skin flap blood supply obstacles; (3) the interosseous dorsal nerve and blood vessels after concomitant downward between forearm bone, should look straight into the sharpness, separation of blood vessel bundle nerve preservation in situ don't damage especially in proximal separation of blood vessel bundle so as not to cause refers to dysfunction.

\section{Conclusion}

Before the really group applied to clinical prevention, with the vascular pedicle flap is more effective method of the repair of hand skin defect, soft tissue defect, keep body length. If you can accord to the provided information for repairing hand wounds with vascular pedicle tissue flap translocation of patients to provide care, we believe that it will achieve the satisfying effect.

\section{Acknowledgement}

This research was financially supported by the National Science Foundation. 


\section{References}

[1] David A. Lombardi, Gary S. Sorock, Leslie Holander, Murray A. Mittleman. A Case-Crossover Study of Transient Risk Factors for Occupational Hand Trauma by Gender[J]. Journal of Occupational and Environmental Hygiene. 2012 (10)

[2] Petr Hyza, Jiri Vesely, Igor Stupka, Emanuele Cigna, Nicola Monni. The Bilobed Arterialized Venous Free Flap for Simultaneous Coverage of 2 Separate Defects of a Digit[J]. Annals of Plastic Surgery. 2012 (6)

[3] Harald E.T. Moshammer, Franz X. Schwarzl, Franz M. Haas, Heinrich Maechler, Gerhard Pierer, Marco Wiltgen, Horst Koch. Retrograde arterialized venous flap: An experimental study[J]. Microsurgery. 2013 (2)

[4] Shohei Omokawa, Yasuhito Tanaka, Jaiyoung Ryu, Nina Clovis. Anatomical Consideration of Reverse-Flow Island Flap Transfers from the Midpalm for Finger Reconstruction[J]. Plastic and Reconstructive Surgery. 2012(7)

ho. Fingertip Reconstruction Using a Volar Flap Based on the Transverse Palmar Branch of the Digital Artery[J]. Annals of Plastic Surgery. 2013 (3)

[6] Daping Yang, Steven F. Morris. Vascular basis of dorsal digital and metacarpal skin flaps[J]. Journal of Hand Surgery. 2012 (1)

[7] R. Adani,R. Busa,R. Scagni,A. Mingione. The Heterodigital Reversed Flow Neurovascular Island Flap for Fingertip Injuries[J]. Journal Of Hand Surgery. 2012(4)

[8] Ahmet Karacalar,Mesut Özcan. A new approach to the reverse dorsal metacarpal artery flap[J]. Journal of Hand Surgery. 2013(2)

[9] Massimo Del Bene, Maurizio Petrolati,Piero Raimondi, Carlo Tremolada, Alex Muset.

Reverse Dorsal Digital Island Flap[J]. Plastic and Reconstructive Surgery. 2012 (3)

[10] P Valenti, AC Masquelet, T Bégué. Anatomic basis of a dorso-commissural flap from the 2nd, 3rd and 4th intermetacarpal spaces[J]. Surgical and Radiologic Anatomy. 2012 (4) 